Document downloaded from:

http://hdl.handle.net/10251/153837

This paper must be cited as:

Broatch, A.; Novella Rosa, R.; Garcia Tiscar, J.; Gómez-Soriano, J. (2019). On the shift of acoustic characteristics of compression-ignited engines when operating with gasoline partially premixed combustion. Applied Thermal Engineering. 146:223-231.

https://doi.org/https://doi.org/10.1016/j.applthermaleng.2018.09.089

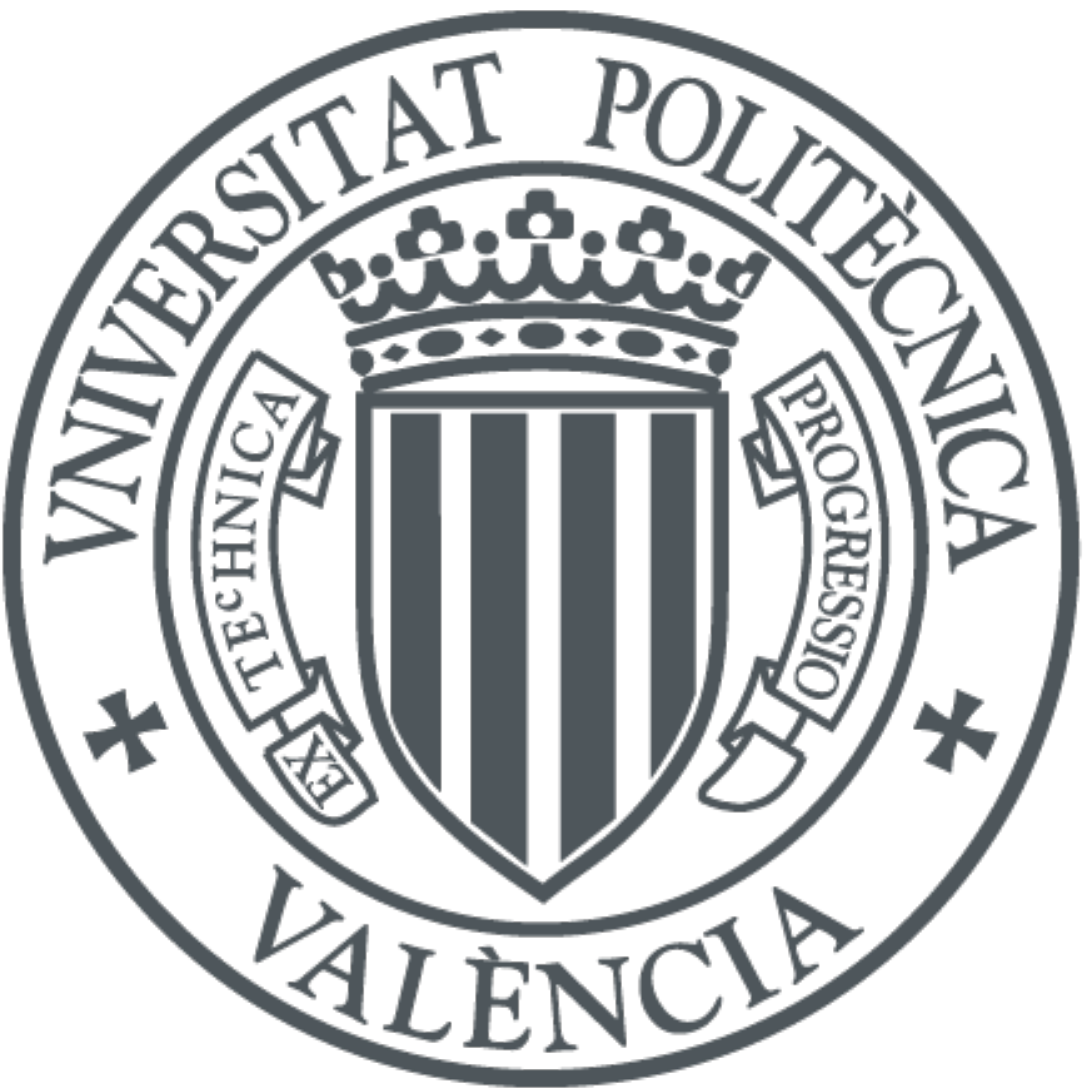

The final publication is available at

10.1016/j.applthermaleng.2018.09.089

Copyright Elsevier

Additional Information 


\title{
On the shift of acoustic characteristics of compression-ignited engines when operating with gasoline partially premixed combustion
}

\author{
A. Broatch ${ }^{\mathrm{a}}$, R. Novella ${ }^{\mathrm{a}, *}, \mathrm{~J}_{\text {. García-Tíscar }}^{\mathrm{a}}, \mathrm{J}$. Gomez-Soriano ${ }^{\mathrm{a}}$ \\ ${ }^{a}$ CMT - Motores Térmicos, Universitat Politècnica de València, Camino de Vera, 46022 Valencia, Spain
}

\begin{abstract}
As the focus of research and development is put into innovative combustion concepts with the goal of reducing harmful chemical pollutants while keeping or even improving the efficiency of conventional Diesel combustion, it is necessary to consider the impact on noise pollution brought by those innovative concepts. However, the question arises as to what extent the noise characterization and optimization strategies currently applied to conventional Diesel compression-ignited engines are applicable to these innovative combustion concepts. In this paper, we apply experimental noise characterization techniques based on pressure trace decomposition to two compression-ignited engines: a baseline conventional Diesel engine and an innovative 2-stroke engine operating with the gasoline partially premixed combustion concept. Analysis of the results reveals that the underlying physical phenomena responsible for the spectral signature of the noise are still shared between the new and the conventional concepts. However, results evince a significant change in the relevance of these physical sources, leading to necessary changes in optimization strategies for future compression-ignited engine development.
\end{abstract}

Keywords: Gasoline PPC concept, Combustion noise, 2-stroke engine, Noise, vibration \& harshness, Resonance

\section{Introduction}

In a context of increased climate change concerns, compression-ignited (CI) engines operating with conventional Diesel combustion (CDC) have been favoured due to their higher efficiency. A crucial factor in reducing the carbon footprint of the transportation section, efficiency of CDC engines comes at the cost of higher emissions of pollutants such as nitrous oxides $\left(\mathrm{NO}_{\mathrm{x}}\right)$ and particulate matter $(\mathrm{PM})$, with a demonstrated impact on public health [1].

As a consequence, intense research efforts have been conducted to develop innovative combustions concepts such as homogeneous charge compression ignition (HCCI) or premixed charge compression ignition (PCCI) which aim to preserve efficiency while reducing pollutant emissions [2-4], thereby enabling cleaner vehicles with a reduced carbon footprint.

However, sustainability and environmental friendliness do not only depend on chemical emissions. Noise pollution has been identified by the World Health Organization as the second leading environmental health hazard [5], besides the aforementioned chemical pollutants. Moreover, road transport is the leading cause of noise, with combustion noise being the primary source at low speeds typical of urban environments.

It is thus imperative to ensure that innovative combustion concepts that are focused on improving emissions do not increase the already hazardous noise levels of modern cities. In this paper, we assess the applicability of current CI noise characterization and optimization strategies, developed for CDC engines, to an innovative 2-stroke engine operating with gasoline partially premixed combustion (PPC) which has demonstrated the capacity to achieve extremely low pollutant emissions, while maintaining or even improving the thermal efficiency $[6,7]$.

This combustion concept operates between completely premixed and fully diffusive conditions, whereby low soot emissions may be attained [8] while retaining the control of nitrous oxides $\left(\mathrm{NO}_{\mathrm{x}}\right)$ with large amounts of Exhaust Gas Recirculation (EGR) [9]. Despite these potential benefits, there are some drawbacks inherent to combustion that still remain unsolved, compromising its application to commercial vehicles. Among others, it is not possible to achieve an accurate control of the combustion phasing through the injection since the extreme thermodynamic conditions required to ensure the ignition of the charge, specifically in terms of temperature, are very particular and difficult to reach with conventional engine devices. In this situation, a 2-stroke engine combined with a Variable Valve Timing (VVT) allows to manage the residual gas fraction within the chamber, thereby controlling the in-cylinder temperature $[6,10]$.

However, despite the advantages of this platform, the fuel consumption operating with gasoline PPC at mediumlow loads is higher if it is compared with CDC operation $[7,11]$. In addition, further studies $[7,12]$ revealed a tradeoff trend between $\mathrm{NO}_{\mathrm{x}}$ emissions and combustion stability when optimizing the operating settings.

*Corresponding author, email: rinoro@mot.upv.es 
The main issue lies probably in the combustion noise exhibited at high loads. Being an inherently noisy combustion mode, mainly due to its high burning rates, noise levels emitted by the engine are excessively high at full load conditions, exceeding by more than $15 \mathrm{~dB}$ levels seen in CDC [13].

Studies conducted in the '60s showed that combustion noise in CDC is generated by the pressure instabilities induced by combustion itself [14-16]. In addition to this pure pressure unsteadiness, the generated pressure waves resonate inside the chamber [17], acting as an extra excitation source [18, 19]. It is unknown if the contribution of these sources to the engine acoustic signature is equal in CDC and PPC [13].

In order to analyze how the acoustic behaviour of the gasoline PPC concept differs from that of current Diesel CI engines, the noise emission of an in-production CDC engine was measured across its most typical operating conditions. The obtained noise map was compared with that of PPC, identifying the most critical points and conducting further analysis on them to gain additional insight into the differences in spectral signature of both combustion modes. Finally, a simple parametric study has been performed to highlight the potential of combustion-related settings optimization in innovative PPC concepts.

In the following sections of this paper, the methodology, its validation and the obtained results are presented. In Section 2 the methodology is described in detail. Section 3 is dedicated to the description of the two experimental facilities used for the noise characterisation. Section 4 discusses the results obtained with the outlined method. Finally, Section 5 summarizes the main conclusions drawn from this study.

\section{Methodology}

In this section, a complete description of the noise characterisation methodology for CDC is provided. This methodology is the result of the most relevant findings over the last fifty years in the combustion noise research, starting from Priede [15] and ending with the latest advances on this topic [20]. The target of this method is to relate the physical noise generation mechanisms to the acoustic response of combustion.

In order to decompose CI combustion noise into its physical constituents, the raw pressure trace of each cycle is processed in this work following the technique already pioneered by Priede [15] and further extended by Payri et al. [21]. This is done, first, by subtracting the low-frequency "pseudomotored pressure trace", exclusively caused by the compressionexpansion process itself.

Although this signal can be theoretically obtained, it was measured in this investigation by switching off fuel injection solely in the studied cylinder during one isolated cycle while the combustion was kept in the other cylinders [21]. This procedure was repeated ten times through the 50 recorded cycles, thereby allowing to estimate an average pseudo-motored signal whereas the operating conditions were maintained.

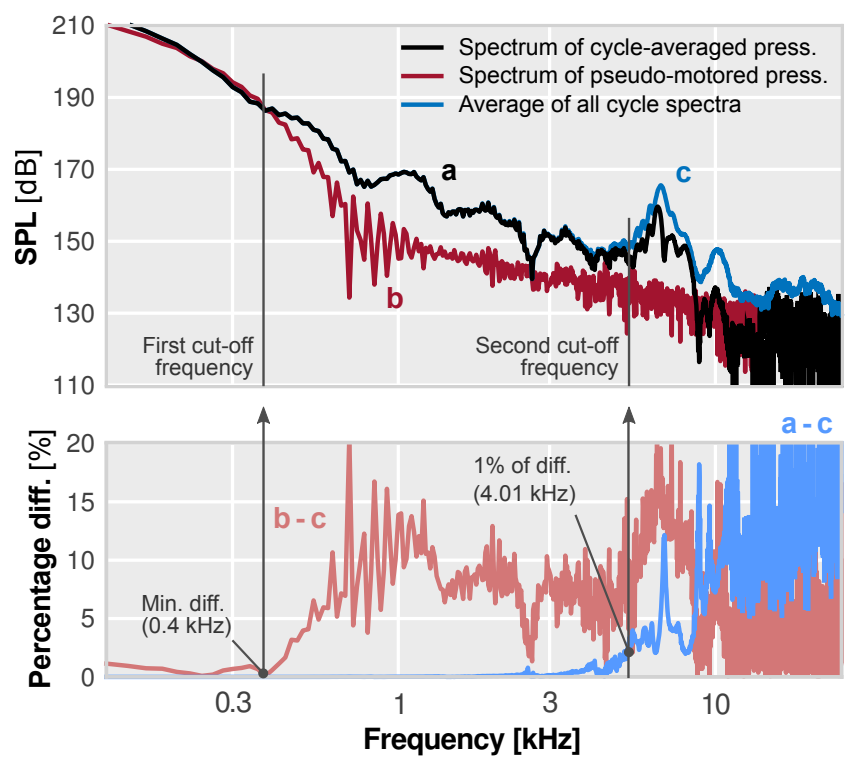

Figure 1: Visual representation of the frequency cut-off determination for an operating condition of the baseline $C D C$ engine, following the selected pressure decomposition algorithm.

Once that this purely mechanical contribution is subtracted, the "excess" pressure signal that remains is then related to the combustion process [22, 23]. From the point of view of the frequency, now it is possible to determine a cut-off frequency between both signals. As it can be seen in Fig. 1, in which data from a given operating condition is plotted as example, the spectrum of pseudo-motored signal (given in Sound Pressure Level, SPL) practically matches the averaged spectrum in the low frequency range. The difference among both traces labelled "b-c" in the bottom graph of this figure, reveals that the minimum gap is around $0.4 \mathrm{kHz}$ and therefore this point was considered as the first cut-off frequency.

An additional difference must be established however between the homogeneous pressure rise caused by the heat release of the combustion, and the resonant gas oscillations inside the chamber caused by the reflections of the pressure waves $[18,19]$.

This resonance-dominant part of the pressure spectra can be isolated through a high-pass filter, the cut-off frequency of which can be determined following the method suggested by Strahle [24] for Diesel engines, where the spectrum of the cycle-averaged pressure trace (instantaneous mean of all recorded cycles) is compared to the average of the spectra of all cycles.

When a significant difference appears between both traces at a certain frequency, then this can be regarded as the start of the frequency range dominated by the resonant pressure wave oscillations. As can be seen in Fig. 1 again, this frequency was determined by the point at which the gap between both traces exceeds $1 \%$ of difference. 
The result of applying this decomposition procedure to the experimental engine data can be seen in Fig. 2. In the top plot, the in-cylinder pressure trace is shown. Raw pressure from a selected test is divided in its corresponding components: mechanical compression, mean combustion pressure, and chamber resonant oscillations.

Cyclical dispersion has been included in these curves by shading representing the standard deviation at each crank angle. Moreover, the frequency spectra of these signals is included in the middle plot of this figure in order to easily see the cause-effect relationship among temporal and frequency domains.

Adding a further step, the spectrum of the sound pressure radiated by the engine block can be estimated from the in-cylinder pressure by applying the approach proposed by Austen and Priede [14] and the engine structural attenuation curve obtained by Anderton [25]. As it can be seen in the bottom plot of Fig. 2, the radiated spectrum can be separated in the same previously delimited three frequency regions, but it is now clear how the resonant peak can dominate the acoustic signature of the engine.
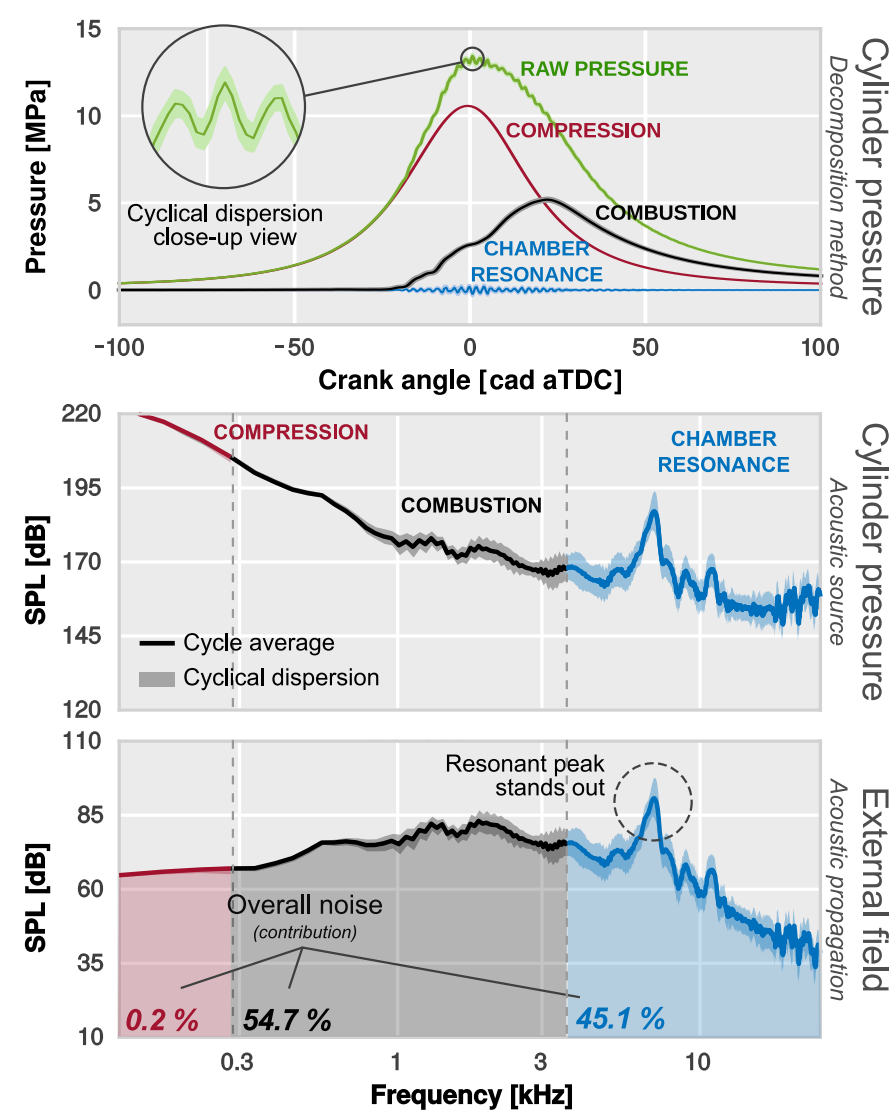

Figure 2: Example of the pressure decomposition technique applied to the baseline $C D C$ engine. In-cylinder pressure is drawn along with each contribution in the time domain (top plot), frequency content (middle plot) and estimated radiated sound pressure levels (bottom plot).
It is also possible to obtain the overall amplitude of each band of frequencies to determine which are their respective contributions to the overall radiated noise. In order to do this, one must recall that the overall noise level of a given operating condition can be obtained by

$$
\mathrm{ON}(\mathrm{dB})=10 \cdot \log _{10} \frac{P_{N}^{2}}{p_{0}^{2}}
$$

where $p_{0}$ is the reference sound pressure of $20 \mu \mathrm{Pa}$, which corresponds to the minimum human hearing threshold. Besides, $\mathrm{P}_{\mathrm{N}}$ is defined as

$$
P_{\mathrm{N}}=\frac{1}{\mathrm{~N}} \cdot \sum_{f=f_{1}}^{f_{\mathrm{N}}} P(f)
$$

where $\mathrm{N}$ is the number of harmonics between $f_{1}$ and $f_{\mathrm{N}}$, and $\mathrm{P}(f)$ represents the Fast Fourier Transform (FFT) of the engine radiated signal, which is obtained by subtracting the structural attenuation curve from the in-cylinder pressure spectrum following Austen's approach [14].

Then, ON is converted to a linear scale. Repeating this procedure for each one of the bounded regions, their overall amplitudes can be obtained. Thus, the ratio between these values expresses the contribution of each band of frequencies to the overall noise, thereby revealing which part of the spectrum is conditioning the noise emission to a greater extent.

Recalling the bottom plot of Fig. 2, the contribution of each band of frequencies is labelled in percentage of the overall noise level. It can be seen that while the low frequency range represents only a negligible part of the ON level $(<1 \%)$, the main contribution is thus shared between the medium ( $\sim$ $55 \%)$ and high frequency regions ( 45\%).

This fact reveal two distinct strategies to act on the emission source. On the one side, the combustion can be modified through the operating settings (injection parameters, EGR, etc.) to reduce the contribution of the middle frequency region [26]. On the other side, the resonant gas oscillations inside the chamber due to reflections of the pressure waves can be mitigated by modifying the combustion-related hardware design [27, 28].

Available literature on combustion noise characterization $[17,26]$ shows that resonant behaviour only depends on the geometric design of the chamber, thus being independent from the combustion law. Therefore, it is possible to decrease, at least theoretically, the resonance intensity and, subsequently, the overall noise emissions, without any remarkable change in the combustion process.

This fact has been exploited by CI engine designers, by first optimizing the combustion law through its different settings, and then the noise signature with slight modifications of the chamber geometry designed to reduce resonant peaks [28]. It remains to be seen if this decoupled strategy can be applied to innovative combustion concepts such as gasoline PPC, and hence the need to test the decomposition technique in both modes. 


\section{Experimental setup}

Since the engines and facilities used in this investigation have been described in previous publications, only the essential information of the experimental facilities and engine configurations will be presented in this section, focusing on the specific data required by this study. Full details about the experimental facilities can be found in the works of Torregrosa, Monelleta et al. [18, 29] and Benajes, Lima et al $[7,8,30]$.

The engines were conscientiously selected for dealing with current design tendencies of the automotive industry for compression-ignited engines in order to contribute to combustion noise fundamental knowledge in automotive engine applications.

\subsection{Baseline engine (Conventional Diesel Combustion)}

The experimental characterization of Diesel combustion was carried out in a light-duty DI Diesel engine. This is a 1.6 l, four-cylinder, turbo-charged engine equipped with a common rail injection system. All tests were performed with a 6-nozzle injector with hole diameters of $124 \mu \mathrm{m}$ and an included spray angle of $150^{\circ}$.

The combustion chamber has a conventional head design with a re-entrant bowl geometry. This piston design provides a geometric compression ratio of 18 . As a reference, the main specifications of the engine and the injector are presented in Table 1.

Table 1: Main specifications and injection system characterisation of the Diesel engine.

\begin{aligned} & \hline Engine type HSDI CI Diesel engine \\ & \hline Fuel [-] Conventional Diesel \\ & Number of cylinders [-] 4 in line \\ & Displacement [cm $\left.{ }^{3}\right] 1600 \\ &$ Bore - Stroke [mm] $75.0-88.3 \\ &$ Connecting rod length [mm] 13.7 \\ & Compression ratio [-] $18: 1 \\ &$ Number of valves [-] 2 intake and 2 exhaust \\ & Injector nozzles [-] 6 \\ & Nozzle holes diameter [ $\mu \mathrm{m}] 124 \\ &$ Included spray angle [deg] 150 \\ & \hline\end{aligned}

The gear box was assembled with the engine block in order to maintain the vibration patterns as close as possible to real operation. In addition, an asynchronous electric brake was directly coupled with the engine for controlling the engine speed. The latter was physically and acoustically isolated with sound damping panels so as to prevent possible interferences in the engine noise measurements. The test bench was installed inside of an anechoic chamber located at laboratory $5 \mathrm{~K}$ of Universitat Politècnica de València, which offers free-field conditions for frequencies above $100 \mathrm{~Hz}$.

In-cylinder pressure was measured with a Kistler 6055Bsp piezoelectric transducer placed in the middle of the intake valves through a glow plug adaptor. Since this kind of sensor installation may add some high-frequency disturbances in the pressure signal due to resonances within the gap between the adapter and the mounting bore, a custom glow plug adapter with reduced gap length (at cost of reduced life of the sensor) was designed, ensuring that natural frequencies of the cavity were beyond $35 \mathrm{kHz}$ and thus that acoustic measurements such as those reported in this paper could be performed free of disturbances. Pressure signals were sampled using a dedicated acquisition system and recorded during 50 consecutive engine cycles. All signals were also registered with a sample frequency of $50 \mathrm{kHz}$, ensuring [31] an effective free-aliasing bandwidth (25 kHz approx.) similar to the human hearing domain $-20 \mathrm{~Hz}$ to $20 \mathrm{kHz}$ - and keeping the maximum effective frequency below the natural frequencies of the sensor adapter.

On the engine operation map, the region framed in the lowmedium speed and low-medium load is traditionally accepted as the most critical operation range since it is the region in which the engine operates most frequently under standard driving conditions. Moreover, usual and future homologation driving cycles are specifically focused on this region [32].

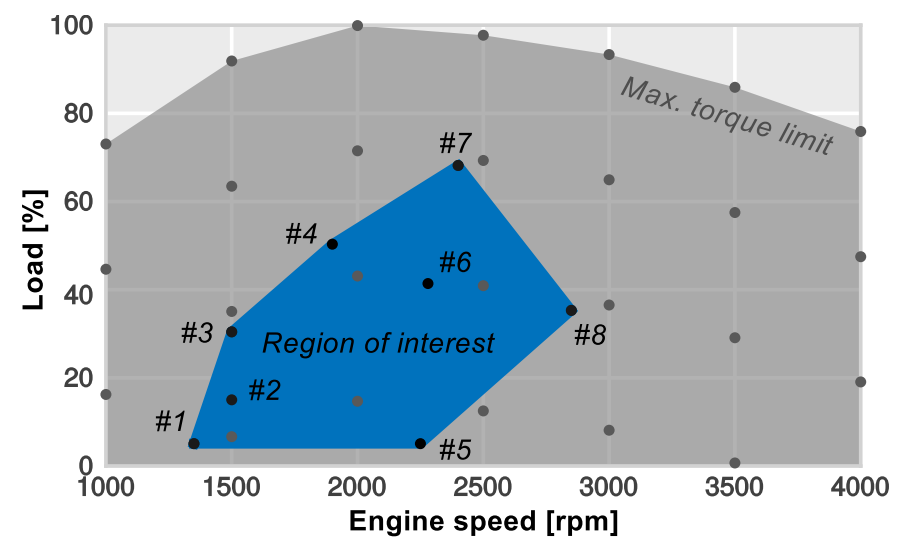

Figure 3: Operating points chosen for the noise characterization analysis of CDC in the baseline engine.

Hence, the region highlighted in Fig. 3 was chosen to quantify both the overall noise emissions and the relevance of the resonance. In this figure, black dots, accompanied by their corresponding number, correspond with the operating conditions used in this study and shown in Table 2, in which the main running settings are summarized.

\subsection{Research engine (Partially Premixed Combustion)}

In this case, experimental activities have been carried out in a single-cylinder research version of an innovative engine concept characterized by a 2-stroke HSDI CI engine with poppet valves scavenge loop. As a reference, the main specifications of this engine are included in Table 3.

A particular design of the cylinder head, represented in Fig. 4, was adopted to optimize the scavenging of burnt gases while keeping short-circuit losses as small as possible during the 2-stroke cycle. The cylinder head geometry exhibits a staged roof for baffling the air flow between intake and ex- 
Table 2: Main running settings of the operation points considered for the noise characterization of CDC.

\begin{tabular}{|c|c|c|c|c|}
\hline Point & $\# 1$ & \#2 & \#3 & \#4 \\
\hline Engine speed [rpm] & 1350 & 1500 & 1500 & 1900 \\
\hline Torque $[\mathrm{Nm}]$ & 12.3 & 36.9 & 75.2 & 123.9 \\
\hline Number Injections [-] & \multicolumn{4}{|c|}{3 (2 pilots + main $)$} \\
\hline Injection pressure [MPa] & 40 & 65 & 87 & 105 \\
\hline Intake pressure [MPa] & 0.104 & 0.107 & 0.118 & 0.161 \\
\hline Point & \#5 & \#6 & \#7 & \#8 \\
\hline Engine speed [rpm] & 2250 & 2280 & 2400 & 2850 \\
\hline Torque $[\mathrm{Nm}]$ & 12.7 & 102.1 & 168.3 & 87.0 \\
\hline Number Injections [-] & \multicolumn{4}{|c|}{3 ( 2 pilots + main $)$} \\
\hline Injection pressure [MPa] & 40 & 95 & 80 & 92 \\
\hline Intake pressure [MPa] & 0.111 & 0.168 & 0.206 & 0.181 \\
\hline
\end{tabular}

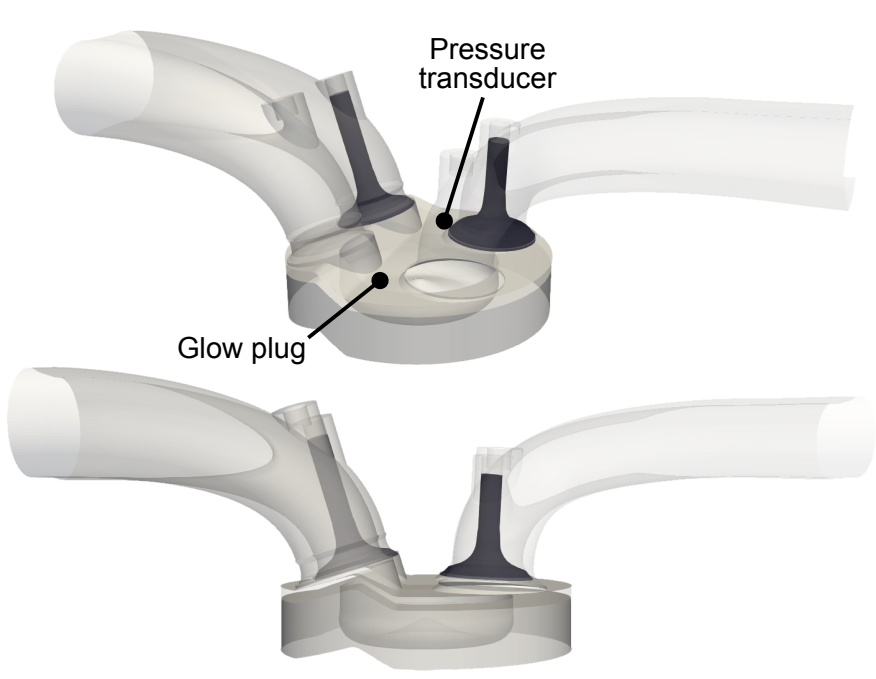

Figure 4: Geometry of the research engine combustion chamber, pipes and valve heads, indicating the positions of the pressure transducer and the glow plug.

haust valves, directing the air to the cylinder wall toward the bottom of it. This geometry represents the best compromise between scavenging efficiency, acceptable permeability, and favourable combustion chamber design [33].

Table 3: Engine specifications and injection system characterization of the 2-Stroke engine.

\begin{aligned} & \hline Engine type 2-stroke HSDI CI engine \\ & \hline Fuel [-] RON95 gasoline \\ & Number of cylinders [-] 1 \\ & Displacement [cm $\left.{ }^{3}\right] 365 \\ &$ Bore - Stroke [mm] $76.0-80.5 \\ &$ Connecting rod length [mm] 14.3 \\ & Compression ratio (geometric) $17.8: 1 \\ &$ Compression ratio (effective) From 13.0:1 to 8.8:1 \\ & Number of valves [-] 2 intake and 2 exhaust \\ & Type of scavenge Poppet valves with scavenge loop \\ & Injector nozzles [-] 8 \\ & Nozzle holes diameter [ $\mu \mathrm{m}] 90 \\ &$ Included spray angle [deg] 118 \\ & \hline\end{aligned}

The timing control of the four poppet valves actuated by double-overhead camshafts relies on a hydraulic cam-driven VVT system, which allows delaying both intake and exhaust valve timings up to +30 degrees from the base cam phasing. This system is well adapted for modifying the air management characteristics, due to its flexibility to adjust the overlap period between intake and exhaust, but also the effective compression and effective expansion ratios.

The engine is equipped with a common rail prototype fuel injection system, which grants a maximum rail pressure of 1100 bar when operates with gasoline fuel. All tests were performed with a nozzle configuration of 8 holes of $90 \mu \mathrm{m}$ of diameter, and with an included angle of $148^{\circ}$. The piston bowl geometry employed was of a conventional design, optimized for operating with the Conventional Diesel Combustion (CDC) concept, and provided a geometric compression ratio of 17.8.

The engine was assembled in a fully instrumented test cell equipped with all auxiliary devices required for engine operation. An external compressor supplied the intake air and simulated the boost conditions. The exhaust back-pressure produced by the turbine was generated and controlled by means of a throttle valve located downstream of the exhaust settling chamber. The installation also included independent water and oil cooling circuits, and an additional low EGR system to provide arbitrary levels of cooled exhaust gas even at very high intake boost pressures.

In-cylinder pressure was measured with a Kistler 6061B pressure transducer placed between the intake and exhaust valves opposite to the glow plug location, while a different piezo-resistive pressure sensor was used for referencing this pressure signal. In this case, the sensor was directly installed at the cylinder head surfaces (flush-mounted) in order to avoid the high-frequency disturbances in the pressure signal commented above. Instantaneous high frequency signals, such as cylinder pressure were sampled using a dedicated acquisition system and recorded during 100 engine cycles for each operation condition. These signals were also registered with a sample frequency of $45 \mathrm{kHz}$, ensuring a free-aliasing bandwidth similar to the human hearing domain.

The most relevant combustion parameters, such as maximum pressure time-derivative, maximum cylinder pressure, rate of heat release and combustion phasing angles were calculated from the in-cylinder pressure by means of the energy equation and some simplifications [34, 35]. This equation was solved assuming uniform pressure and temperature throughout the whole combustion chamber volume and yielded the instantaneous mean temperature and the heat release.

Although being a single-cylinder engine particularly designed for research purposes, an operating region equivalent to that considered in the previous section with the CDC study has been measured. Therefore, twelve tests were carried out with the specifications shown in Table 4. 
Table 4: Main running settings of the operation points considered for the noise characterization of gasoline PPC concept.

\begin{tabular}{rllllll}
\hline Point & $\mathbf{\# \mathbf { 1 } ^ { \prime }}$ & $\mathbf{\# \mathbf { 2 } ^ { \prime }}$ & $\mathbf{\# \mathbf { 3 } ^ { \prime }}$ & $\mathbf{\# \mathbf { 4 } ^ { \prime }}$ & $\mathbf{\# \mathbf { 5 } ^ { \prime }}$ & $\mathbf{\# \mathbf { 6 } ^ { \prime }}$ \\
\hline Engine speed [rpm] & 1250 & 1250 & 1250 & 1500 & 1500 & 1500 \\
IMEP [MPa] & 0.31 & 0.55 & 1.04 & 0.10 & 0.31 & 0.55 \\
Number Injections [-] & 2 & 2 & 3 & 2 & 2 & 3 \\
Injection pressure [MPa] & 40 & 60 & 85 & 30 & 40 & 60 \\
Intake pressure [MPa] & 0.150 & 0.170 & 0.276 & 0.125 & 0.150 & 0.180 \\
\hline Point & $\mathbf{\# 7 ^ { \prime }}$ & $\mathbf{\# \mathbf { 8 } ^ { \prime }}$ & $\mathbf{\# \mathbf { 9 } ^ { \prime }}$ & $\mathbf{\# 1 0}$ & $\mathbf{\# 1 \mathbf { 1 } ^ { \prime }}$ & $\mathbf{\# 1 2}$ \\
\hline Engine speed [rpm] & 1500 & 1750 & 1750 & 1750 & 2000 & 2500 \\
IMEP [MPa] & 1.04 & 0.31 & 0.55 & 1.04 & 0.31 & 0.55 \\
Number Injections [-] & 3 & 2 & 3 & 2 & 3 & 3 \\
Injection pressure [MPa] & 85 & 40 & 60 & 40 & 80 & 60 \\
Intake pressure [MPa] & 0.275 & 0.150 & 0.170 & 0.150 & 0.180 & 0.170 \\
\hline & & & & & &
\end{tabular}

\section{Results and discussion}

In this section, results from the methodology application are presented and discussed. First, the methodology is applied to several points within the operating map of the CDC production engine. Subsequently, this application is extended to the research engine operating with gasoline PPC concept.

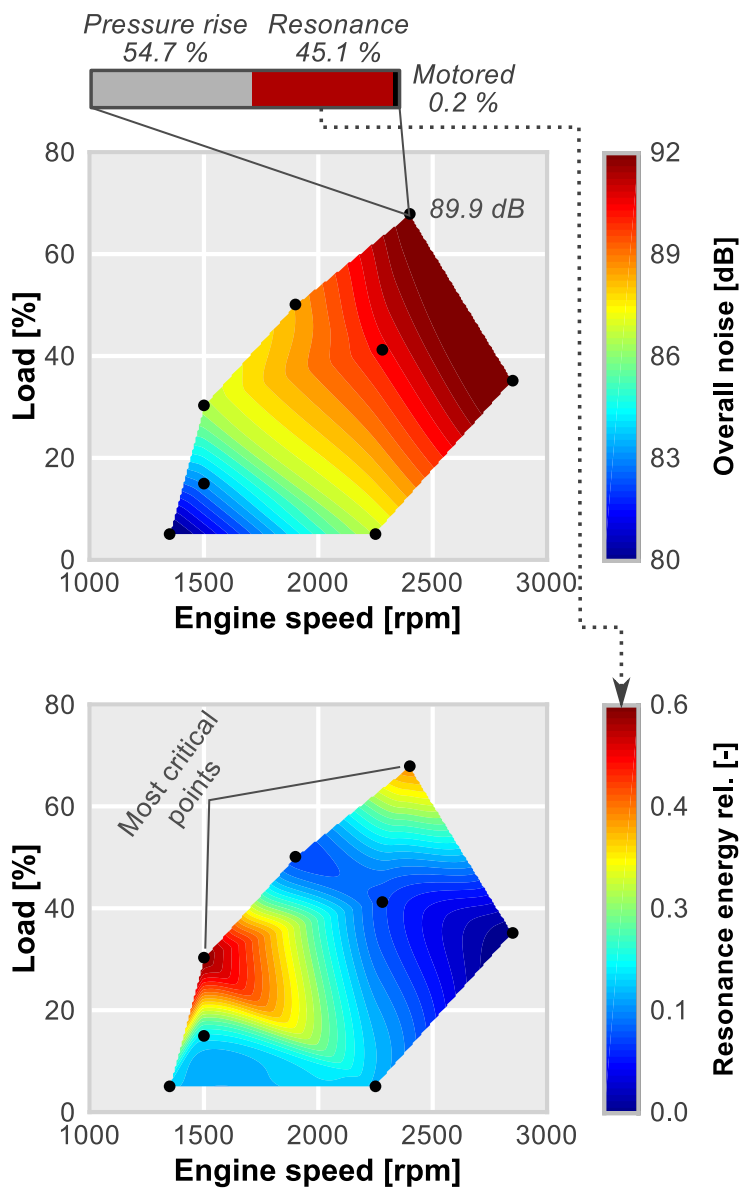

Figure 5: Noise and resonance contribution maps for CDC operation. The overall noise and the contribution of resonance energy maps are interpolated and plotted using contour plots.
Finally, further analysis is presented taking into account other injection settings and additional results with CDC mode in the research engine to ensure a fair comparison between the acoustic signature of both combustion concepts.

\subsection{Conventional Diesel Combustion}

Besides the identification of the region of interest, the metrics related to the noise characterization were included in Fig. 5 using a cubic polynomial fit for the visualization. As can be seen from contours plotted on the top graph, ON levels monotonically increase with the engine speed and also with the load, evincing that the most serious issues are focused on the top-right region of the map considered.

However, the relevance of the resonance is not correlated with the engine speed neither the load, as is shown by the contours plotted on the bottom graph of the previously referred figure. The colour scale shows the contribution of the resonant oscillations, this is, the overall amplitude of the high frequency content isolated above.

Thus, as can be seen in the stacked bar plot included in this figure, a value of 0.45 means that the ON level is $45 \%$ influenced by the resonant frequency content and $55 \%$ affected by the medium range of frequencies. Note that the contribution of the low range of frequencies is not considered here due to its small significance $(<1 \%)$.

It becomes readily apparent that different mechanisms are influencing the noise generation due to combustion as the operating point is changing along the map. For instance, noise generation is clearly dominated by the resonant phenomena within the zone of lower engine speeds and loads gathered by 50 and $100 \mathrm{Nm}$ of torque whereas the highest speed region (point \#8) is mostly controlled by the homogeneous pressure rise rate due to combustion, which covers the middle frequency range.

Aside from these opposed zones, there are other points in which a resonance lowering could mean a significant noise reduction. In this sense, point \#7 is the most interesting since a small resonance lowering could significantly reduce the high levels of ON exhibited. 


\subsection{Gasoline Partially Premixed Combustion}

Owing to the clear propensity to generate extremely high burning rates, Low Temperature Combustion (LTC) concepts are inherently noisy and the acoustic emission is an important constraint for their application to commercial vehicles. The study presented in the previous section is expanded here to the gasoline PPC mode described in [36-39], being an interesting concept from the point of view of both emissions and efficiency and enough representative of noise issues shown by new LTC concepts.

Figure 6 displays the results obtained from gasoline PPC measurements. Following a similar representation of Fig. 5, the overall noise level is shown at top and the resonance relevance at the bottom. As can be seen, combustion noise is now highly correlated with the engine load rather than the engine speed. Apart from this, noise levels are considerably higher if they are compared with CDC; points with low acoustic levels exhibit around $90 \mathrm{~dB}$ in gasoline PPC whereas their corresponding points in CDC rarely exceed $81 \mathrm{~dB}$.

Regarding the resonance contribution, it is not correlated with the operating parameters plotted in the graph, showing an arbitrary pattern along the measured map as in Diesel combustion. However, some significant differences are also observed; the most evident, the maximum values of the res-
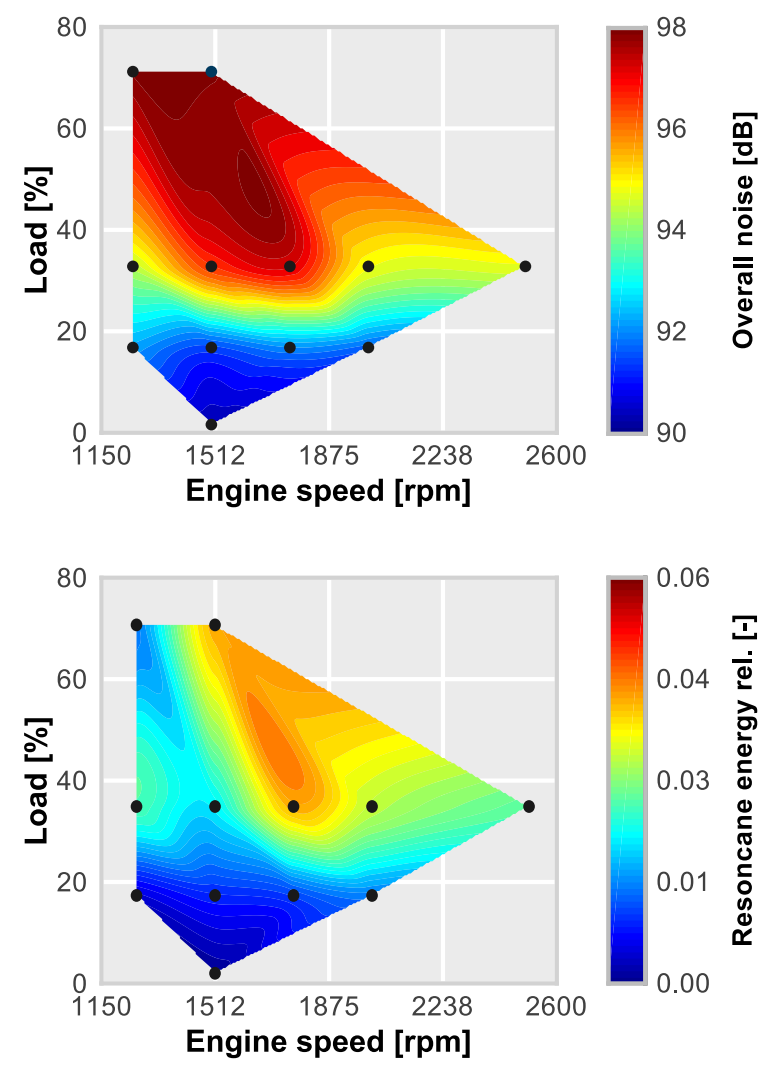

Figure 6: Noise and resonance contribution maps for gasoline PPC operation. The overall noise and the contribution of resonance energy maps are interpolated and plotted using contour plots. onance contribution. While these values reached $45 \%$ in several Diesel operating points, they do not surpass $5 \%$ in the whole gasoline PPC map, thereby suggesting that resonant constitution in this combustion concept is almost negligible.

In order to extent the scope of the analysis, point \#7' was selected to carry out additional measurements since it has been shown as the most unfavourable operating point from the point of view of noise emissions. Moreover, previous works $[7,10]$ revealed that the main injection timing, commonly refereed by the start of energizing (SoE) of the injector, has the most notable impact on combustion features. Therefore, an additional study was performed by sweeping $\mathrm{SoE}_{\text {main }}$ each 2 cad between -42 and -34 cad aTDC with the settings of point \#7'.

Once the measurements were finished, a frequency analysis was performed with the aim of finding significant differences. Results are shown in Fig. 7 (top), in which the average spectrum of all measured tests are plotted together for comparison. An extra test was carried out in the research engine operating with CDC. It is important to note that this test was carried out in the research engine with optimized settings in order to provide the same outputs to easily and fairly compare both combustion modes in the same engine platform, avoiding possible uncertainties due to operating points or engine architecture differences.

As it can be seen, all PPC tests exhibit very similar SPL traces, with only a few differences in the middle range of frequencies $(0.2-2.9 \mathrm{kHz})$. This gap becomes notably larger if we compare PPC with CDC, but mostly in the medium frequency band. Resonant amplitudes are very similar in both combustion modes, demonstrating the independence of this wave propagation phenomenon from the combustion process itself.

In the bottom plot of Fig. 7, the externally-radiated sound spectra has been estimated through Anderton's method. It is evident that the spectral signature in PPC mode is dominated by broadband noise, in contrast with CDC where tonal and narrow band noise is more prominent.

This becomes specially notorious if we compare these spectral signatures with those of the baseline CDC engine shown in Fig. 2. In that case, the frequency content of the noise was dominated by a clear resonant peak; in PPC mode however the broadband content overcomes the resonance peaks by $\sim 20 \mathrm{~dB}$.

In consequence, even though PPC presents higher ON levels, its noise quality, which is influenced by the spectral signature, is also different from CDC. While a psychoacoustic evaluation of this quality is outside the scope of this investigation, it remains an important factor to consider in future studies.

Returning to the parametric study, Fig. 8 is included to analyse the influence of $\mathrm{SoE}_{\text {main }}$ in $(\mathrm{d} p / \mathrm{d} t)_{\max }$, resonance energy $\left(E_{r e s}\right)$ and $O N$. Resonance energy characterizes the global amplitude of the pressure oscillations due to the resonance 


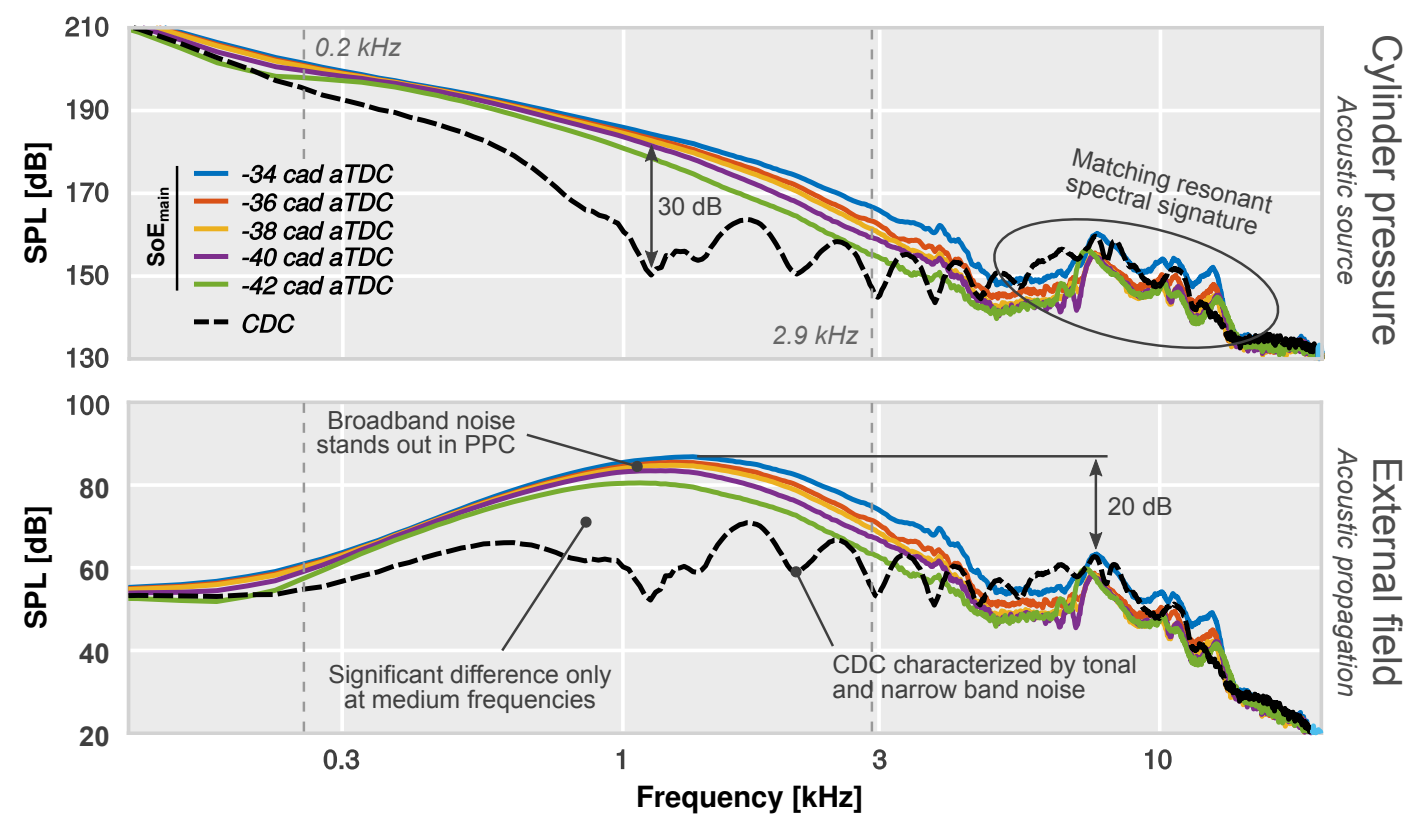

Figure 7: Frequency analysis of gasoline PPC concept sweeping the timing of the main injection. In-cylinder pressure spectra comparison is plotted at the top whereas the externally-radiated spectral signature predicted by the Anderton method is shown at the bottom.

phenomenon and is defined [40] as:

$$
\mathrm{E}_{\mathrm{res}}=\int_{\mathrm{IVC}}^{\mathrm{EVO}} p(t)_{\mathrm{res}}^{2} \mathrm{~d} t
$$

where $p(t)_{\text {res }}$ is the resonance pressure signal that results from the decomposition procedure, which is integrated between intake valves closing (IVC) and exhaust valves opening (EVO).

As these graphs show, all parameters tend to increase as the $\mathrm{SoE}_{\text {main }}$ is delayed towards the TDC. However, maximum pressure rise rate and $\mathrm{ON}$ increase monotonically, whereas the energy of resonance evolution tends to be exponential. Inspecting the ON plot (center), we observe however that it is very similar to that of $(\mathrm{d} p / \mathrm{d} t)_{\max }$, highlighting the tight correlation between the two parameters and the small influence of resonance in PPC.
The comparison of these results with those obtained with CDC shows that the significant differences observed in the medium range of frequencies are related to the important variations in the pressure rise rates. When operating with gasoline PPC, $(\mathrm{d} p / \mathrm{d} t)_{\max }$ is one order of magnitude larger compared to that obtained when operating with $\mathrm{CDC}$ even at earlier values of $\mathrm{SoE}_{\text {main }}$.

Extremely high burning rates generated by gasoline PPC combustion explain this fact, as indeed the pressure rise rate is strongly influenced by this parameter [41]. On the contrary, resonance levels are reasonably similar until a certain $\mathrm{SoE}_{\text {main }}$ ( -36 cad aTDC). However, additional shifts in $\mathrm{SoE}_{\text {main }}$ towards the TDC result in a notable increase of the resonant energy.
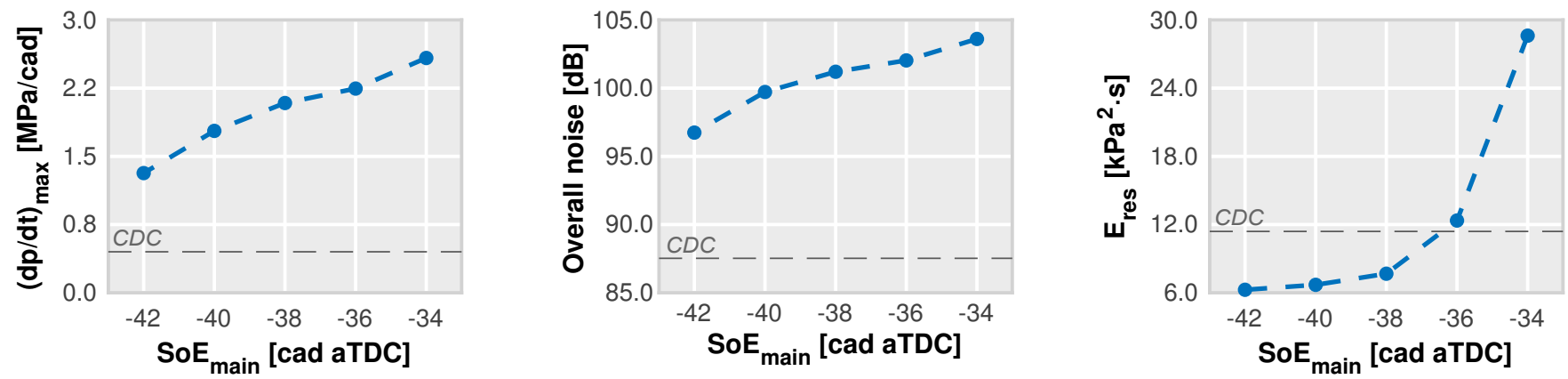

Figure 8: Influence of $S o E_{\text {main }}$ on maximum pressure rise rate, overall noise resonance energy. The threshold for all three variables obtained operating the research engine with optimized settings in CDC mode is also included for comparison. 


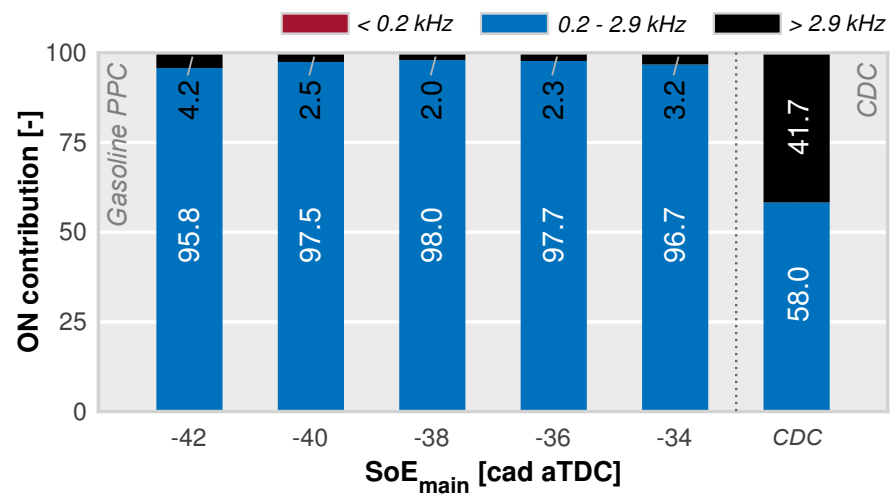

Figure 9: Contribution of each bandwidth of frequencies to ON level estimation, including a measurement of the research engine operating in CDC mode.

It seems clear at this point that the lower contribution of resonance to the ON levels is a consequence of the higher pressure rise rates achieved during the combustion rather than a reduction of the resonance, as suggested early in Fig. 7. This fact can be demonstrated by applying again the method for determining the contribution of each frequency band to the total ON.

Figure 9 compares the contribution of each frequency band to the calculated ON level in all $\mathrm{SoE}_{\text {main }}$ sweep tests. CDC test is also included for reference. The extremely different contribution of medium-high frequency bands in both combustion concepts is evident.

As it can be seen, the contribution of the resonance phenomena to the engine noise is again here almost negligible $(<5 \%)$ when operating with gasoline PPC while modifying the operating settings, thereby pointing to the maximum pressure rise rate as the major influencing parameter in engine noise radiation. Consequently, results displayed in Fig. 6 can be considered representative of the engine operation.

On the other hand, the proportions demonstrated by CDC in the research engine are more comparable and, in fact, very similar to those observed in the baseline production engine (shown at the top plot of Fig. 5), with both cases indicating an approximate $60-40 \%$ balance. This demonstrates that the acoustic relevance of the different physical phenomena are dictated fundamentally by the combustion mode and not by the particular engine design.

In view of the main limitations of the gasoline PPC concept already summarized in the introduction, it is interesting to analyse whether previously observed trends are reproducing and what role noise outputs play on them. In this way, values of $\mathrm{NO}_{\mathrm{x}}$ emissions and combustion efficiency [39] are plotted in Fig. 10 for each case considered in the $\mathrm{SoE}_{\text {main }}$ parametric study. As can be seen from this figure, the trade-off already observed by other authors $[7,12]$ is also evinced in this study: the combustion stability is deteriorated as $\mathrm{NO}_{\mathrm{x}}$ are reduced.
Recalling the overall noise trend drawn in Fig. 8, it can be seen that $\mathrm{NO}_{\mathrm{x}}$ and noise emissions follow the same tendency: their values decrease as the main injection is performed earlier. Thus, a new trade-off among noise emissions and combustion efficiency arises, compromising again the applicability of the concept.

\section{Conclusions}

In this study, the same CI combustion noise characterization technique based on in-cylinder pressure decomposition has been implemented in two engines; a baseline production CDC engine and a research engine able to employ innovative combustion concepts. In both cases, the analysis was extended to different operating conditions. In light of the results, the following conclusions can be drawn:

- In the CDC case, mid-frequency, broadband combustion noise dictated by $(\mathrm{d} p / \mathrm{d} t)_{\max }$ and resonant noise characterized by higher frequency peaks can contribute equally to ON levels, with resonant peaks dominating the spectral signature of the noise.

- Analysis of several operating points revealed that the balance between the contribution of the two phenomena in the CDC case is highly dependent on the operating conditions, with the resonance accounting for up to $60 \%$ of the ON in some cases, with ON levels varying in the 80-90 dB range.

- ON levels in the gasoline PPC were generally higher than in the CDC case, with levels between 90-98 dB across the map. Therefore, the benefits of PPC in terms of efficiency and emissions could be lowered by its impact on noise, for both the passengers and the urban environment.

- While resonance levels in the research engine were similar for PPC and CDC modes, their contribution to ON levels was much lower in PPC. PPC noise was unquestionably dominated by the medium band of frequencies which is, in turn, dictated by the maximum $(\mathrm{d} p / \mathrm{d} t)_{\max }$. Even so, the proposed methodology correctly estimated cut-off points between the different physical phenomena.

- The methodology allowed to identify a new trade-off between noise emissions and combustion stability when operating by gasoline PPC, thereby contributing to increase the knowledge about PPC operation.

In sum, even though that $\mathrm{CI}$ combustion noise characterization techniques based on spectral pressure decomposition have demonstrated their validity in the case of an innovative concept such as PPC, the difference in terms of contribution of the physical phenomena to the ON levels shown in this investigation indicates a need of shifting the optimization strategy for these combustion modes. 

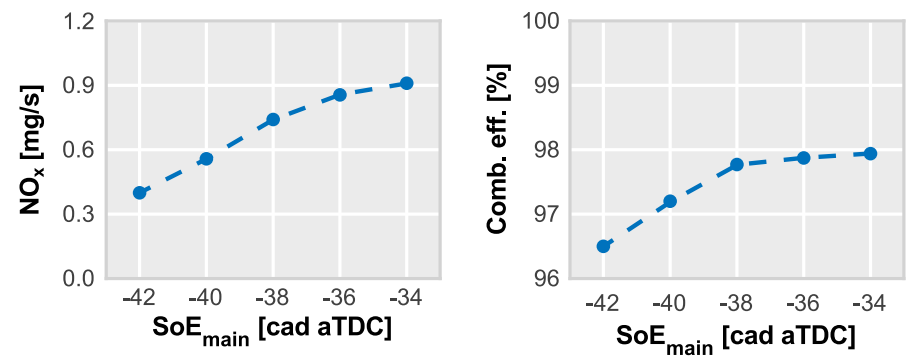

Figure 10: Influence of $\mathrm{SoE}_{\text {main }}$ on $\mathrm{NO}_{\mathrm{x}}$ emissions and combustion efficiency.

While up until now a crucial factor in optimizing the noise emission of CDC engines was the reduction of wave resonance through the design of the combustion chamber geometry, this factor is of little importance in PPC noise. Thereby, acoustic design of PPC engines needs to be focused instead on the precise selection of combustion parameters, introducing ON targets in the merit functions of the optimization algorithms from the beginning.

In a positive note, however, results show that the same decomposition methodology can still be applied successfully in both concepts. Furthermore, the parametric study has demonstrated an influence of almost $1 \mathrm{~dB}$ per degree of $\mathrm{SoE}_{\text {main }}$ advance, highlighting the potential of these parameters for optimizing adverse noise emissions.

\section{Acknowledgements}

The equipment used in this work has been partially supported by FEDER project funds "Dotación de infraestructuras científico técnicas para el Centro Integral de Mejora Energética y Medioambiental de Sistemas de Transporte (CiMeT)" [grant number FEDER-ICTS-2012-06], framed in the operational program of unique scientific and technical infrastructure of the Spanish Government. J. Gomez-Soriano is partially supported through the Programa de Apoyo para la Investigación y Desarrollo (PAID) of Universitat Politècnica de València [grant number FPI-S2-2016-1353].

\section{References}

[1] M. Kampa, E. Castanas, Human health effects of air pollution, Environmental Pollution 151 (2) (2008) 362-367, proceedings of the 4th International Workshop on Biomonitoring of Atmospheric Pollution (With Emphasis on Trace Elements). doi: 10.1016/j .envpol . 2007.06.012.

[2] H. Ryo, Y. Hiromichi, HCCI Combustion in a DI Diesel engine, SAE Technical Paper 2003-01-0745. doi : 10 . 4271/2003-01-0745.

[3] T. W. Ryan, T. J. Callahan, Homogeneous Charge Compression Ignition of Diesel Fuel, SAE Technical Paper 961160. doi: 10.4271/961160.

[4] A. J. Torregrosa, A. Broatch, A. García, L. F. Mónico, Sensitivity of combustion noise and NOx and soot emissions to pilot injection in PCCI Diesel engines, Applied Energy 104 (0) (2013) 149-157. doi: $10.1016 / j$. apenergy . 2012 . 11.040.

[5] World Health Organization, Burden of disease from environmental noise: Quantification of healthy life years lost in Europe, Copenhage, Dinamarca, 2011.

[6] J. Benajes, A. García, V. Domenech, R. Durrett, An investigation of partially premixed compression ignition combustion using gasoline and spark assistance, Applied Thermal Engineering 52 (2) (2013) 468-477. doi: 10.1016/j . applthermaleng. 2012.12.025.
[7] J. Benajes, R. Novella, D. De Lima, P. Tribotte, Investigation on multiple injection strategies for gasoline PPC operation in a newly designed 2-stroke HSDI compression ignition engine, SAE International Journal of Engines 8 (2015-01-0830) (2015) 758-774. doi: $10.4271 / 2015-01-0830$.

[8] J. Benajes, S. Molina, R. Novella, D. De Lima, Implementation of the Partially Premixed Combustion concept in a 2-stroke HSDI diesel engine fueled with gasoline, Applied Energy 122 (2014) 94-111. doi:10. $1016 / j$. apenergy . 2014.02.013.

[9] W. L. Hardy, R. D. Reitz, A Study of the Effects of High EGR, High Equivalence Ratio, and Mixing Time on Emissions Levels in a HeavyDuty Diesel Engine for PCCI Combustion, SAE Technical Paper 200601-0026. doi: 10.4271/2006- 01 - 0026.

[10] J. Benajes, R. Novella, D. De Lima, P. Tribotté, N. Quechon, P. Obernesser, V. Dugue, Analysis of the combustion process, pollutant emissions and efficiency of an innovative 2-stroke HSDI engine designed for automotive applications, Applied Thermal Engineering 58 (1) (2013) 181-193. doi:10.1016/j . applthermaleng . 2013.03.050.

[11] J. Benajes, J. Martin, R. Novella, D. De Lima, Analysis of the load effect on the partially premixed combustion concept in a 2-stroke HSDI diesel engine fueled with conventional gasoline, SAE Technical Paper 2014-01-1291. doi: 10.4271/2014-01-1291.

[12] J. Benajes, R. Novella, D. D. Lima, K. Thein, Impact of injection settings operating with the gasoline Partially Premixed Combustion concept in a 2-stroke HSDI compression ignition engine, Applied Energy 193 (2017) 515-530. doi:10.1016/j . apenergy .2017.02 .044.

[13] A. Broatch, X. Margot, R. Novella, J. Gomez-Soriano, Combustion noise analysis of partially premixed combustion concept using gasoline fuel in a 2-stroke engine, Energy 107 (2016) 612-624. doi:10.1016/j . energy. 2016.04.045.

[14] A. E. W. Austen, T. Priede, Origins of diesel engine noise, SAE Technical Paper 590127. doi: 10.4271/590127.

[15] T. Priede, Relation between form of cylinder-pressure diagram and noise in diesel engines, Proceedings of the Institution of Mechanical Engineers: Automobile Division 14 (1) (1960) 63-97. doi:10.1243/ pime_auto_1960_000_012_02.

[16] T. Priede, E. C. Grover, Paper 2: Noise of Industrial Diesel Engines, Proceedings of the Institution of Mechanical Engineers, Conference Proceedings 181 (3) (1966) 73-89. doi:10.1243/pime_conf_1966_ 181_062_02.

[17] R. Hickling, D. A. Feldmaier, S. H. Sung, Knock-induced cavity resonances in open chamber Diesel engines, The Journal of the Acoustical Society of America 65 (5) (1979) 1474-1479. doi : 10.1121/1.382910.

[18] A. J. Torregrosa, A. Broatch, J. Martín, L. Monelletta, Combustion noise level assessment in direct injection Diesel engines by means of incylinder pressure components, Measurement Science and Technology 18 (7) (2007) 2131-2142. doi: 10.1088/0957-0233/18/7/045.

[19] A. J. Torregrosa, A. Broatch, X. Margot, V. Marant, Combustion chamber resonances in direct injection automotive Diesel engines: a numerical approach, International Journal of Engine Research 5 (1) (2003) 83-91. doi: $10.1243 / 146808704772914264$.

[20] A. Broatch, R. Novella, R. García-Tíscar, Gomez-Soriano, Experimental characterization of combustion noise in compression-ignited engines, Submited to Applied Thermal Engineering. 
[21] F. Payri, A. Broatch, B. Tormos, V. Marant, New methodology for incylinder pressure analysis in direct injection diesel engines - application to combustion noise, Measurement Science and Technology 16 (2) (2005) 540-547. doi : 10. 1088/0957-0233/16/2/029.

[22] J. Yang, R. W. Anderson, A new criterion for judging SI engine incylinder pressure development for its effect on combustion noise, Journal of Engineering for Gas Turbines and Power 120 (3) (1998) 664-668. doi:10.1115/1.2818197.

[23] J. Chung, J. Oh, M. Sunwoo, A real-time combustion control with reconstructed in-cylinder pressure by principal component analysis for a CRDI diesel engine, Journal of Engineering for Gas Turbines and Power 139 (6) (2017) 062802. doi:10.1115/1.4035395.

[24] W. C. Strahle, Combustion randomness and diesel engine noise: theory and initial experiments, Combustion and Flame 28 (1977) 279-290. doi : 10. 1016/0010-2180 (77) 90033-5.

[25] D. Anderton, Relation between combustion system and engine noise, SAE Technical Paper 790270. doi : 10.4271/790270.

[26] A. J. Torregrosa, A. Broatch, X. Margot, J. Gomez-Soriano, Towards a Predictive CFD Approach for Assessing Noise in Diesel Compression Ignition Engines. Impact of the Combustion Strategies, International Conference on Modeling and Diagnostics for Advanced Engine systems: the 9th COMODIA, Okayama (Japan).

[27] A. J. Torregrosa, A. Broatch, J. García-Tíscar, J. Gomez-Soriano, Modal decomposition of the unsteady flow field in compression-ignited combustion chambers, Combustion and Flame 188 (2018) 469-482. doi: 10.1016/j . combustflame. 2017.10.007.

[28] A. Broatch, R. Novella, J. Gomez-Soriano, P. Pal, S. Som, Numerical Methodology for Optimization of Compression-Ignited Engines Considering Combustion Noise Control, SAE International Journal of Engines 2018-01-0193. doi : 10.1016/2018-01-0193.

[29] L. Monelletta, Contribution to the study of combustion noise of automotive Diesel engines, Ph.D. thesis, Universitat Politècnica de València (2010).

[30] D. de Lima, Analysis of combustion concepts in a poppet valve twostroke downsized compression ignition engine designed for passenger car applications, Ph.D. thesis, Universitat Politècnica de València (2016).
[31] F. Ihlenburg, The Medium-Frequency Range in Computational Acoustics: Practical and Numerical Aspects, Journal of Computational Acoustics 11 (02) (2003) 175-193. doi : 10.1142/S0218396X03001900.

[32] F. Steinparzer, P. Nefischer, W. Stütz, The future of the diesel engine as a means of propulsion in passenger cars, 15th Internationales Stuttgarter Symposium: Automobilund Motorentechnik (2015) 1363-1380doi: 10. 1007/978-3-658-08844-6_95.

[33] P. Tribotte, F. Ravet, V. Dugue, P. Obernesser, N. Quechon, J. Benajes, R. Novella, D. De Lima, Two Strokes Diesel Engine - Promising Solution to Reduce CO2 Emissions, Procedia - Social and Behavioral Sciences 48 (2012) 2295-2314. doi : 10.1016/j . sbspro.2012.06.1202.

[34] M. Lapuerta, O. Armas, J. J. Hernández, Diagnosis of DI Diesel combustion from in-cylinder pressure signal by estimation of mean thermodynamic properties of the gas, Applied Thermal Engineering 19 (5) (1999) 513-529. doi : 10.1016/S1359-4311 (98)00075- 1.

[35] F. Payri, P. Olmeda, J. Martín, A. García, A complete 0D thermodynamic predictive model for direct injection diesel engines, Applied Energy 88 (12) (2011) 4632-4641. doi : 10 . 1016/j . apenergy . 2011.06.005.

[36] R. Hanson, D. Splitter, R. Reitz, Operating a Heavy-Duty Direct-Injection Compression-Ignition Engine with Gasoline for Low Emissions, SAE Technical Paper 2009-01-1442. doi : 10.4271/2009-01- 1442.

[37] V. Manente, B. Johansson, P. Tunestal, W. Cannella, Effects of Different Type of Gasoline Fuels on Heavy Duty Partially Premixed Combustion, SAE International Journal of Engines 2009-01-2668 (2009) 71-88. doi: 10.4271/2009-01- 2668.

[38] A. Broatch, X. Margot, R. Novella, J. Gomez-Soriano, Impact of the injector design on the combustion noise of gasoline partially premixed combustion in a 2-stroke engine, Applied Thermal Engineering 119 (2017) 530-540. doi:10.1016/j .applthermaleng.2017.03.081.

[39] A. Broatch, R. Novella, J. García-Tíscar, J. Gomez-Soriano, Potential of dual spray injectors for optimising the noise emission of gasoline partially premixed combustion in a 2-stroke HSDI CI engine, Applied Thermal Engineering (2018) 369-378doi:10.1016/j.applthermaleng. 2018.01. 108.

[40] A. Broatch, X. Margot, A. Gil, C. Donayre, Computational study of the sensitivity to ignition characteristics of the resonance in DI diesel engine combustion chambers, Engineering Computations 24 (1) (2007) 77-96. doi : 10. 1108/02644400710718583.

[41] S. Rulik, W. Wróblewski, G. Nowak, J. Szwedowicz, Heat transfer intensification using acoustic waves in a cavity, Energy 87 (0) (2015) 21-30. doi : 10.1016/j . energy . 2015.04.088. 